\title{
Virtual Reference Feedback Tuning for two degree of freedom controllers
}

\author{
A.Lecchini* ${ }^{*}$ M.C. Campi**(1) and S.M. Savaresi*** \\ ${ }^{*}$ CESAME - Université Catholique de Louvain \\ Avenue Georgés Lemaitre 4, B-1348 Louvain-la-Neuve, Belgium \\ lecchini@auto.ucl.ac.be \\ ${ }^{* *}$ Dip. di Elettronica per l'Automazione - Università di Brescia \\ Via Branze 38, 25123 Brescia, Italy \\ campi@ing.unibs.it \\ *** Dip. di Elettronica ed Informazione - Politecnico di Milano \\ Piazza Leonardo da Vinci 32, 20133 Milano, Italy \\ savaresi@elet.polimi.it
}

\begin{abstract}
The Virtual Reference Feedback Tuning (VRFT) is a data based method for the design of feedback controllers. In the original formulation, the VRFT method gives a solution to the one degree of freedom model-reference control problem in which the objective is to shape the input-output transfer function of the control system. In this paper, the extension of the method to the design of two degree of freedom controllers is presented and discussed.
\end{abstract}

(1) Corresponding author 


\section{Introduction}

In this paper we consider the problem of designing a controller for a plant, when no mathematical description of the plant dynamical behavior is available.

It is common experience in industrial control design that a mathematical description of the plant to be controlled is not available and that undertaking a modeling study is considered too costly and time-consuming. In these cases, one would like to come up with a controller tuned directly from measurements coming from the plant without going through a modeling phase.

One method that has been developed to this purpose is the so-called Virtual Reference Feedback Tuning (VRFT) algorithm. Precisely, VRFT permits one to tune a controller within a specified class on the basis of a single set of I/O (Input/Output) data collected from an experiment on the plant. The idea on which VRFT is based was originally proposed in [17] and subsequently developed in $[10,9,24]$ as a complete and ready-to-use method. In $[10,9,24]$ the VRFT method has been presented for the selection of 1 degree of freedom (d.o.f.) controllers. In this case, one single controller transfer function fed by an error signal (that is the difference between the reference and the measured plant output) determines the control input value. Such a 1 d.o.f. setting permits one to shape one single closed-loop transfer function, and in fact in [10] only the reference to output transfer function was considered. The aim of this paper is to present and discuss the extension of the method to a 2 d.o.f. setting, where both the reference to output and the disturbance to output transfer functions are taken care of.

First, the control problem setting is precisely described. Later on in this section, we discuss the different approaches that have been introduced in the literature in order to address this control problem and put VRFT into perspective with other methods.

\section{Problem statement}

Given a discrete-time SISO (Single Input - Single Output) plant $P(z)$ with input $u(t)$ and output $y(t)$ affected by an additive disturbance signal $d(t)$ :

$$
y(t)=P(z) u(t)+d(t)
$$

consider the 2 d.o.f. control system having the following structure (see Fig.1):

$$
\left\{\begin{array}{l}
y(t)=P(z) u(t)+d(t) \\
u(t)=C_{r}\left(z ; \theta_{r}\right) r(t)-C_{y}\left(z ; \theta_{y}\right) y(t)
\end{array}\right.
$$

where $r(t)$ is the reference signal, and $\left(C_{r}\left(z ; \theta_{r}\right), C_{y}\left(z ; \theta_{y}\right)\right)$ is a 2 d.o.f. controller belonging to a 


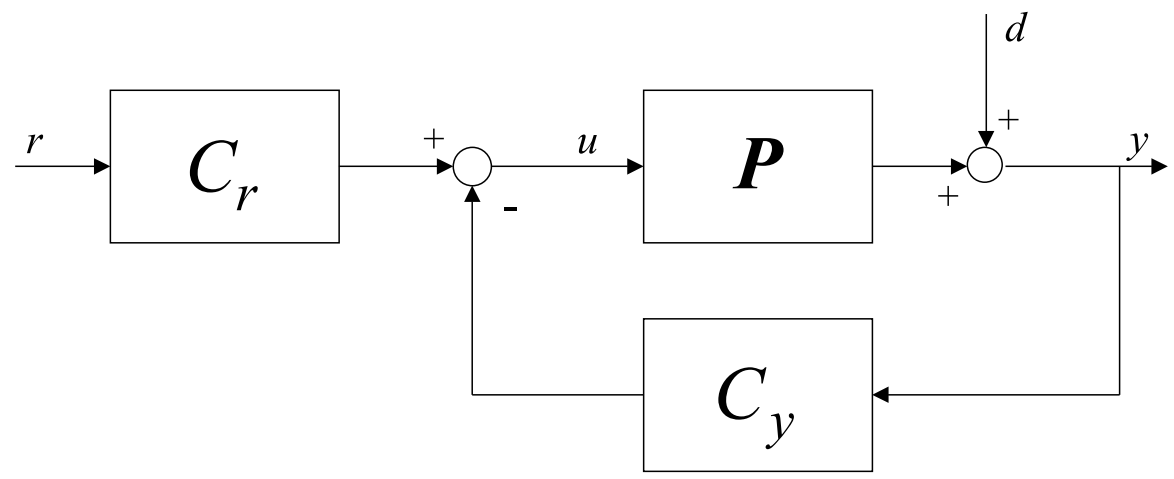

Figure 1: The two degree of freedom control system.

given family of parameterized controllers. If $C_{r}\left(z ; \theta_{r}\right)=C_{y}\left(z ; \theta_{y}\right)$, the 1 d.o.f. controller studied in [10] is recovered (in this case, the controller transfer functions $C_{r}\left(z ; \theta_{r}\right)$ and $C_{y}\left(z ; \theta_{y}\right)$ are usually implemented through a single block placed in the loop). The attention is restricted to linearly parameterized controllers, that is transfer function $C_{r}\left(z ; \theta_{r}\right)$ takes the form $C_{r}\left(z ; \theta_{r}\right)=\beta_{r}(z)^{T} \theta_{r}$ and, similarly, $C_{y}\left(z ; \theta_{y}\right)=\beta_{y}(z)^{T} \theta_{y}$, where $\beta_{r}(z)$ and $\beta_{y}(z)$ are vectors of discrete-time transfer functions (with dimension $n r$ and $n y$, respectively), and $\theta_{r}$ and $\theta_{y}$ are the parameter vectors.

In the sequel, the transfer function between $r(t)$ and $y(t)$ of the control system (2) will be referred to as closed-loop function, and the transfer function between $d(t)$ and $y(t)$ will be named sensitivity function.

Given a reference model $M(z)$ for the closed-loop function, a reference model $S(z)$ for the sensitivity function, and a family of parameterized controllers $\left\{\left(C_{r}\left(z ; \theta_{r}\right), C_{y}\left(z ; \theta_{y}\right)\right)\right\}$, the control problem consists in selecting the controller parameter vectors $\left(\bar{\theta}_{r}, \bar{\theta}_{y}\right)$ which minimizes the following model reference criterion:

$$
J_{M R}\left(\theta_{r}, \theta_{y}\right)=\left\|\left(\frac{P(z) C_{r}\left(z ; \theta_{r}\right)}{1+P(z) C_{y}\left(z ; \theta_{y}\right)}-M(z)\right) W_{M}(z)\right\|_{2}^{2}\left\|\left(\frac{1}{1+P(z) C_{y}\left(z ; \theta_{y}\right)}-S(z)\right) W_{S}(z)\right\|_{2}^{2}
$$

where $W_{M}(z)$ and $W_{S}(z)$ are user-chosen weighting functions.

A point which need be made clear is that minimizing (3) is not a standard optimal control problem, since we assume that the plant transfer function $P(z)$ is not known. In order to compensate for such a lack of knowledge, in the VRFT approach it is assumed that the designer is given a set of I/O measurements collected from the plant $P(z)$ (either in open-loop or in closed-loop). Such a set of data has to be used to come up with a sensible solution to the control problem design without having further access to the plant for experiment. 


\section{Discussion on the control setting}

The problem of designing feedback controllers (usually industrial PID controllers) on the basis of a set of I/O measurements has attracted the attention of control engineers since the 1940s with the pioneering work by Ziegler and Nichols, [35]. After the original work by Ziegler and Nichols, many more techniques have been proposed, exploring different directions (see e.g. [12, 19, 13]). The reader is referred to $[28,2]$ for book-length presentations of these methods.

By comparing VRFT with the commonly used tuning rules for PID controllers presented in [35, 12, 19, 13, 28, 2], some main differences are worth noticing:

- VRFT aims at finding a solution to a model-reference control problem (3), while other methods usually try to find a generically "well-working" solution according to some criterion "built-in" in the method. While this introduces an extra difficulty for the designer (since he/she has to select suitable reference models), it also adds an important degree of freedom in the definition of the control specifications. It should also be noted that $M(z)$ and $S(z)$ can as well be chosen to be very simple transfer functions (of degree 1 or 2) in standard applications.

- VRFT is not restricted to PID controllers. In this paper, we consider the wide class of linearly parameterized controllers, that includes PID controllers as a special case. On the other hand, VRFT also applies to nonlinearly parameterized controllers, at the price of extra algorithmic complications. Moreover, the idea underlying VRFT can be extended so as to cover non-linear controllers as well (see e.g. [29, 30, 18] for a discussion on this).

- The computational effort required in the implementation of VRFT is higher than for standard tuning rules. However, in view of the computational power of modern digital circuits and computers, this does not appear to be a substantial limitation.

The real bottleneck in the design of a data-based controller lies in the number of experiments to perform on the real plant, since these experiments are time consuming and often require to halt the normal operation of the system. In this respect, VRFT is comparable to standard tuning rules as it requires a single data set collected from one single experiment on the plant.

It is also important to mention that, in the field of data-based control system design, many advances have been made in recent years. Almost all these methods, however, share the feature of being iterative, that is they require to perform many experiments on the plant. They can be grouped in indirect (i.e. model-based) methods, [1, 31, 15, 25, 26, 4, 20, 16, 33, 34, 14], and direct methods, $[23,22,32,21]$.

- The indirect iterative methods iteratively perform plant model identification and model-based controller design. The successively selected controllers are applied to the plant and new data 
are generated for the next selection. The idea is that the controller should progressively become better tuned to the actual plant, so attaining increasingly higher performance.

- The direct methods aim at directly finding the controller, without an intermediate identification step.

Among these methods, a very interesting scheme called Iterative Feedback Tuning (IFT) has been recently proposed by Hjalmarsson and coauthors, [23, 22, 21]. IFT is based on a gradient-descent approach and calls for a sequence of experiments on the true plant with specific inputs. As a matter of fact, the brightness of the method relies on a very smart selection of such inputs, in such a way that the gradient can be estimated from the corresponding measured output. IFT returns the (local) optimal controller parameter.

Even if IFT and VRFT belong to the same class of design methods (direct-methods), their peculiar features are quite different. VRFT is a "one-shot" method which searches for the global minimum of the control performance index, with no need for iterations or an initialization. In fact, it uses only one set of input-output data. However, as we shall see, the VRFT technique is only nearlyoptimal, in the sense that, in general, it provides a controller which is close, but not equal, to the one minimizing (3). On the contrary, IFT provides a fine tuning of the controller towards the optimal solution.

In short, we could say that VRFT provides a good solution with little effort, while IFT points to the optimal solution, but is more time-demanding. Thus, we see that the realm of applicability of VRFT is more similar to that of standard tuning methods as described above than the one of IFT. As an additional remark, we also note that VRFT can also be used as a powerful initialization method for iterative techniques.

\section{Closed-loop stability}

It is well known that a model reference problem may lead to an unstable closed-loop system, see e.g. [3] for a study of the conditions under which this happens. In the context of the present contribution, however, the plant is supposed to be unknown, and therefore assessing the validity of these conditions is normally not feasible. Rather, in line with the adopted framework that only a set of measurements is available, one should conceive to use data-based validation tests for stability.

This important aspect is not treated in the present contribution. Yet, we note that data-based stability validation tests have started to appear in the literature, and the reader is referred to $[5,7,6,9,8,11]$ for contributions in this direction.

The outline of this paper is as follows. In Section 2 the "Virtual Reference" idea for the two degree of freedom setting is introduced, the design algorithm is described, and an analysis of the achievable performance is given. For the sake of clarity, in Section 2 it is assumed that the data are 
not corrupted by noise (i.e. $d(t)=0$ ). The use of noisy data is discussed in Section 3. A simulation example, in Section 4, ends the paper.

\section{The Virtual Reference algorithm for the design of two degree of freedom controllers}

We commence by introducing the basic idea underlying the VRFT approach for 2 d.o.f. controllers. It extends in a natural way that for the 1 d.o.f. case.

\section{The Virtual Reference idea}

Suppose that, for a certain given controller, the control system (2) happens to have the closed-loop transfer function $M(z)$ from $r(t)$ to $y(t)$. Then, if the closed-loop system is fed by any reference signal $r(t)$, and $d(t)=0$, its output equals $y(t)=M(z) r(t)$. Thus, by concentrating on a specific given reference $\bar{r}(t)$, we can say that a necessary condition for the closed-loop system to have the same closed-loop transfer function as the reference model $M(z)$ is that the output of the two systems is the same for that given reference.

In model reference control, the latter condition is typically imposed by first selecting $\bar{r}(t)$ and then by choosing the controller such that this condition is in fact satisfied. However, for a generic selection of $\bar{r}(t)$, the above task may turn out to be difficult to accomplish if a model of the plant is not available.

The basic idea behind the virtual reference approach consists in performing a wise selection of $\bar{r}(t)$ so that the determination of the controller becomes easy. Moreover, for 2 d.o.f control schemes, the idea can be extended to the design of the sensitivity function as well.

To be specific, the construction of $\bar{r}(t)$ is now described in detail.

In the beginning, we are given two files of data, the first one containing measurements of the input $u(t)$ and the second one containing measurements of the corresponding output $y(t)$. For the sake of clarity, in the coming discussion we assume that there is no noise, so that $y(t)=P(z) u(t)$. Given the measured $y(t)$, consider a reference $\bar{r}(t)$ such that $M(z) \bar{r}(t)=y(t)$. Such a reference is called "virtual" because it does not exist in reality and it was not used in the actual generation of $y(t)$. On the other hand, it does exist as a file (i.e. it can be generated in our computer based on the equation $M(z) \bar{r}(t)=y(t))$. Notice that $y(t)$ can be interpreted as the desired output of the control system (2) when the reference signal is $\bar{r}(t)$ and $d(t)=0$.

Observe now that, even though plant $P(z)$ is not known, we know that when $P(z)$ is fed by $u(t)$ (the measured input signal contained in the first file that has not been used as far), it generates $y(t)$ as an output. Therefore, we can claim that a good controller $\left(C_{r}\left(z ; \theta_{r}\right), C_{y}\left(z ; \theta_{y}\right)\right)$ (at least in the condition when the reference signal is the virtual reference $\bar{r}(t)$ and $d(t)=0$ ) should generate $u(t)$ when fed by $\bar{r}(t)$ and $y(t)$, because this way the desired output $y(t)$ is obtained at the output of the control system. 


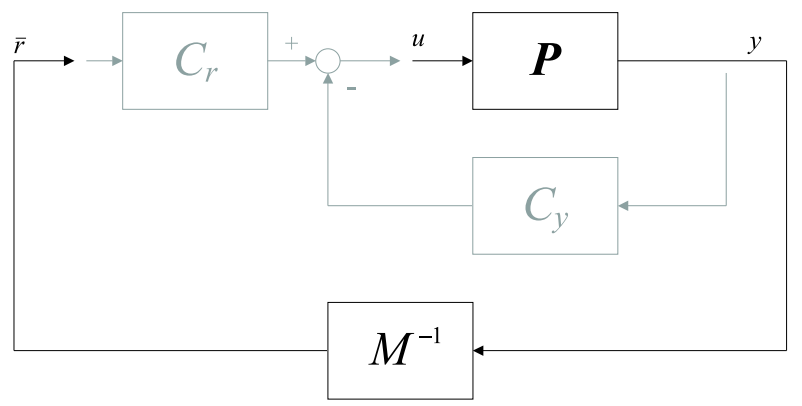

Figure 2: The construction of the virtual reference.

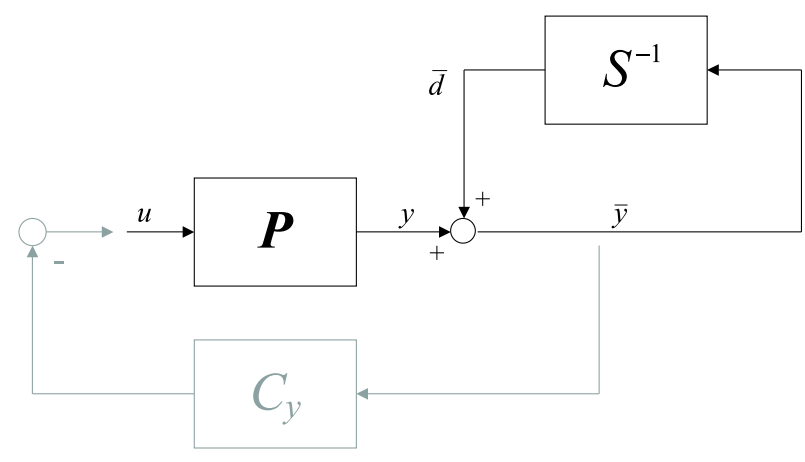

Figure 3: The construction of the virtual disturbance.

The construction of the virtual reference $\bar{r}(t)$ is illustrated in Fig. 2.

A similar reasoning can be applied to the sensitivity function as well.

Given the measured $y(t)$, consider a signal $\bar{d}(t)$ such that $y(t)+\bar{d}(t)$ is the desired output when the reference signal is zero $(r(t)=0)$ and the disturbance signal is $\bar{d}(t)$. Thus, the signal $\bar{d}(t)$ is such that $y(t)+\bar{d}(t)=S(z) \bar{d}(t)$ and this equation can be used for the computation of $\bar{d}(t)$. We can now claim that a good controller $C_{y}\left(z ; \theta_{y}\right)$ (at least in the case in which the disturbance is $\bar{d}(t)$ and $r(t)=0)$ is one that generates $u(t)$ when fed by $\bar{y}(t):=y(t)+\bar{d}(t)$.

The construction of $\bar{d}(t)$ and $\bar{y}(t)$ is illustrated in Fig. 3 .

The above idea is implemented in the following algorithm. In the algorithm, we have also included the prefiltering of data through the filters $L_{M}(z)$ and $L_{S}(z)$. Later on, we shall see that a suitable selection of these filters is important to optimize the algorithm performance.

\section{The design algorithm}

Given the reference models $M(z)$ and $S(z)$, and the set of data $\{u(t), y(t)\}_{t=1, . ., N}$, do the following:

1. Construct:

- $\bar{r}(t)$ such that $y(t)=M(z) \bar{r}(t)$;

- $\bar{d}(t)$ such that $y(t)+\bar{d}(t)=S(z) \bar{d}(t) ;$ and

$-\bar{y}(t)=y(t)+\bar{d}(t)$.

2. Select the controller parameter vectors $\left(\hat{\theta}_{r}^{N}, \hat{\theta}_{y}^{N}\right)$ that minimize the following performance index

$$
J_{V R}^{N}\left(\theta_{r}, \theta_{y}\right)=\frac{1}{N} \sum_{t=1}^{N}\left[L_{M}(z)\left(u(t)-C_{r}\left(z ; \theta_{r}\right) \bar{r}(t)+C_{y}\left(z ; \theta_{y}\right) y(t)\right)\right]^{2}
$$




$$
+\frac{1}{N} \sum_{t=1}^{N}\left[L_{S}(z)\left(u(t)+C_{y}\left(z ; \theta_{y}\right) \bar{y}(t)\right)\right]^{2}
$$

in which $L_{M}(z)$ and $L_{S}(z)$ are suitable filters (initialization problems are neglected here, that is the filtered signals are assumed to be known from the first instant $t=1$ ).

Notice that when $C_{r}\left(z ; \theta_{r}\right)=\beta_{r}(z)^{T} \theta_{r}$ and $C_{y}\left(z ; \theta_{y}\right)=\beta_{y}(z)^{T} \theta_{y}$ (i.e. the controller depends linearly on the parameter vectors) the performance index (4) is quadratic and the parameters $\left(\hat{\theta}_{r}^{N}, \hat{\theta}_{y}^{N}\right)$ are easily obtained by solving the normal equations

$$
\begin{gathered}
{\left[\begin{array}{c}
\hat{\theta}_{r}^{N} \\
\hat{\theta}_{y}^{N}
\end{array}\right]=A_{N}^{-1} F_{N}} \\
A_{N}=\frac{1}{N} \sum_{t=1}^{N}\left(\left[\begin{array}{c}
\varphi_{L_{M}}^{\bar{r}}(t) \\
-\varphi_{L_{M}}^{y}(t)
\end{array}\right]\left[\begin{array}{c}
\varphi_{L_{M}}^{\bar{r}}(t) \\
-\varphi_{L_{M}}^{y}(t)
\end{array}\right]^{T}+\left[\begin{array}{c}
0 \\
\varphi_{L_{S}}^{\bar{y}}(t)
\end{array}\right]\left[\begin{array}{c}
0 \\
\varphi_{L_{S}}^{\bar{y}}(t)
\end{array}\right]^{T}\right) \\
F_{N}=\frac{1}{N} \sum_{t=1}^{N}\left(\left[\begin{array}{c}
\varphi_{L_{M}}^{\bar{r}}(t) \\
-\varphi_{L_{M}}^{y}(t)
\end{array}\right] u_{L_{M}}(t)-\left[\begin{array}{c}
0 \\
\varphi_{L_{S}}^{\bar{y}}(t)
\end{array}\right] u_{L_{S}}(t)\right)
\end{gathered}
$$

where

$$
\begin{aligned}
& \varphi_{L_{M}}^{\bar{r}}(t)=\beta_{r}(z) L_{M}(z) \bar{r}(t) ; \quad \varphi_{L_{M}}^{y}(t)=\beta_{y}(z) L_{M}(z) y(t) ; \quad \varphi_{L_{S}}^{\bar{y}}(t)=\beta_{y}(z) L_{S}(z) \bar{y}(t) ; \\
& u_{L_{M}}(t)=L_{M}(z) u(t) ; \quad u_{L_{S}}(t)=L_{S}(z) u(t)
\end{aligned}
$$

\section{Analysis of the design criterion}

The analysis of the controller design criterion will be conducted by resorting to the notion of ideal controller $\left(C_{r}^{0}(z), C_{y}^{0}(z)\right)$. This is the controller that solves the model matching problem exactly, namely $C_{r}^{0}(z)$ and $C_{y}^{0}(z)$ are given by:

$$
\begin{aligned}
C_{r}^{0}(z) & =\frac{1}{P(z)} \frac{M(z)}{S(z)}, \\
C_{y}^{0}(z) & =\frac{1}{P(z)} \frac{1-S(z)}{S(z)}
\end{aligned}
$$

Notice that $\left(C_{r}^{0}(z), C_{y}^{0}(z)\right)$ in general does not belong to the available family of parameterized controllers $\left\{\left(C_{r}\left(z ; \theta_{r}\right), C_{y}\left(z ; \theta_{y}\right)\right)\right\}$. Even more so, $C_{r}^{0}(z)$ and $C_{y}^{0}(z)$ could as well be improper rational functions, that is, strictly speaking, they are not transfer functions. The notion of ideal controller 
$\left(C_{r}^{0}(z), C_{y}^{0}(z)\right)$ will be used in the following only as an analysis tool.

The analysis of $J_{V R}^{N}\left(\theta_{r}, \theta_{y}\right)$ is based on asymptotic results. Under the hypothesis of ergodicity of the involved signals, using the Parseval theorem (see e.g. [27]) and the definition of $C_{r}^{0}(z)$ and $C_{y}^{0}(z)$, the following asymptotic (as $N \rightarrow \infty$ ) frequency domain representation of $J_{V R}^{N}\left(\theta_{r}, \theta_{y}\right)$ is easily obtained

$$
\begin{aligned}
J_{V R}\left(\theta_{r}, \theta_{y}\right) & =\frac{1}{2 \pi} \int_{-\pi}^{\pi}|P|^{2}\left|C_{r}^{0}-C_{r}\left(\theta_{r}\right)+M\left(C_{y}\left(\theta_{y}\right)-C_{y}^{0}\right)\right|^{2} \frac{\left|L_{M}\right|^{2}}{|M|^{2}} \Phi_{u} d \omega \\
& +\frac{1}{2 \pi} \int_{-\pi}^{\pi}|P|^{2}\left|C_{y}\left(\theta_{y}\right)-C_{y}^{0}\right|^{2} \frac{|S|^{2}\left|L_{S}\right|^{2}}{|S-1|^{2}} \Phi_{u} d \omega
\end{aligned}
$$

where $\Phi_{u}$ is the power spectral density of $u(t)$ (throughout we drop the argument $e^{j \omega}$ in transfer functions). The criterion $J_{V R}\left(\theta_{r}, \theta_{y}\right)$ is the asymptotic counterpart of $J_{V R}^{N}\left(\theta_{r}, \theta_{y}\right)$, namely the criterion to which $J_{V R}^{N}\left(\theta_{r}, \theta_{y}\right)$ tends as the number of available data goes to infinity. Accordingly, as $N \rightarrow \infty$, the minimum $\left(\hat{\theta}_{r}^{N}, \hat{\theta}_{y}^{N}\right)$ of $J_{V R}^{N}\left(\theta_{r}, \theta_{y}\right)$ will converge to the minimum of $J_{V R}\left(\theta_{r}, \theta_{y}\right)$, say $\left(\hat{\theta}_{r}, \hat{\theta}_{y}\right.$ ) (in fact, the latter convergence requires additional assumptions. One possibility is to ask that the minimizer is searched in a compact set and that convergence takes place uniformly). In the following, for analysis purposes, $J_{V R}\left(\theta_{r}, \theta_{y}\right)$ will be extensively used in place of $J_{V R}^{N}\left(\theta_{r}, \theta_{y}\right)$.

Consider now the criterion $J_{M R}\left(\theta_{r}, \theta_{y}\right)$ (which is the original criterion we want to minimize). Using the definition of 2-norm of a discrete-time linear transfer function and the definition of $C_{r}^{0}(z)$ and $C_{y}^{0}(z), J_{M R}\left(\theta_{r}, \theta_{y}\right)$ can be given the following form:

$$
\begin{aligned}
J_{M R}\left(\theta_{r}, \theta_{y}\right) & =\frac{1}{2 \pi} \int_{-\pi}^{\pi}|P|^{2} \frac{\left|C_{r}^{0}-C_{r}\left(\theta_{r}\right)+M\left(C_{y}\left(\theta_{y}\right)-C_{y}^{0}\right)\right|^{2}}{\left|1+P C_{y}\left(\theta_{y}\right)\right|^{2}}\left|W_{M}\right|^{2} d \omega \\
& +\frac{1}{2 \pi} \int_{-\pi}^{\pi}|P|^{2} \frac{\left|C_{y}\left(\theta_{y}\right)-C_{y}^{0}\right|^{2}}{\left|1+P C_{y}\left(\theta_{y}\right)\right|^{2}}|S|^{2}\left|W_{S}\right|^{2} d \omega
\end{aligned}
$$

The minimum point of $J_{M R}\left(\theta_{r}, \theta_{y}\right)$ is indicated in the following by $\left(\bar{\theta}_{r}, \bar{\theta}_{y}\right)$.

By comparing (8) and (9), we note that, if $\left(C_{r}^{0}(z), C_{y}^{0}(z)\right) \in\left(C_{r}\left(z ; \theta_{r}\right), C_{y}\left(z ; \theta_{y}\right)\right)$, then the minimum of $J_{V R}\left(\theta_{r}, \theta_{y}\right)$ corresponds to the ideal controller and coincides with the minimum of $J_{M R}\left(\theta_{r}, \theta_{y}\right)$, whatever the plant, the filters and the reference models are. Therefore, in the ideal case in which the ideal controller belongs to the class of available controllers, the controller estimated through the virtual reference approach coincides with the ideal one.

On the other hand, it is apparent that $J_{V R}\left(\theta_{r}, \theta_{y}\right)$ and $J_{M R}\left(\theta_{r}, \theta_{y}\right)$ have different minima when the class of available controllers has restricted complexity, so that $\left(C_{r}^{0}(z), C_{y}^{0}(z)\right) \notin\left(C_{r}\left(z ; \theta_{r}\right), C_{y}\left(z ; \theta_{y}\right)\right)$. 
It is in this case that the filters $L_{M}(z)$ and $L_{S}(z)$ enter into play and prove their usefulness. As a matter of fact, we show below that, by a suitable selection of these filters, it is possible to match a certain extended virtual reference criterion with the second order expansion of the corresponding extended model reference criterion around its minimizer (see below for details). This way, minimizing the virtual reference criterion leads to a nearly optimal solution for the original model reference problem. Before proceeding, we would also like to mention the main observation that makes such a result possible. The extended model reference criterion and the extended virtual reference criterion exhibit a different dependence on the unknown plant $P(z)$. Yet, their second derivatives around the minimizer of the extended model reference criterion have the same dependence on $P(z)$. This is why suitable $P(z)$-independent filters $L_{M}(z)$ and $L_{S}(z)$ (they must be $P(z)$-independent since $P(z)$ is not known) can be selected so as to obtain the matching.

\section{The choice of the filters}

Introduce the following choice of the filters: select $L_{M}(z)$ and $L_{S}(z)$ in such a way that

$$
\begin{aligned}
\left|L_{M}\right|^{2} & =|M|^{2}|S|^{2}\left|W_{M}\right|^{2} \frac{1}{\Phi_{u}} \\
\left|L_{S}\right|^{2} & =|S-1|^{2}|S|^{2}\left|W_{S}\right|^{2} \frac{1}{\Phi_{u}}
\end{aligned}
$$

Since all the quantities in the right-hand-side of equations (10) and (11) are known, the filters can be actually constructed (in fact, $\Phi_{u}$ may be considered known only when the input signal characteristics are chosen by the designer; otherwise, $\Phi_{u}$ has to be estimated). In Theorem 2.1 below, we show that this choice attains the above described matching result.

Before stating the theorem, some notations are in order. Let

$$
\begin{aligned}
\Delta C_{r}(z) & :=C_{r}^{0}(z)-\beta_{r}^{T}(z) \bar{\theta}_{r} \\
\Delta C_{y}(z) & :=C_{y}^{0}(z)-\beta_{y}^{T}(z) \bar{\theta}_{y}
\end{aligned}
$$

where $\left(\bar{\theta}_{r}, \bar{\theta}_{y}\right)$ is the parameter vector which minimizes $J_{M R}\left(\theta_{r}, \theta_{y}\right)$. Then, define an extended family of controllers

$$
\left\{\left(C_{r}^{+}\left(z ; \theta_{r}^{+}\right)=\beta_{r}^{+}(z)^{T} \theta_{r}^{+}, \quad C_{y}^{+}\left(z ; \theta_{y}^{+}\right)=\beta_{y}^{+}(z)^{T} \theta_{y}^{+}\right)\right\}
$$

where

$$
\begin{aligned}
& \beta_{r}^{+}(z)=\left[\begin{array}{ll}
\beta_{r}(z)^{T} & \Delta C_{r}(z)
\end{array}\right]^{T} ; \quad \beta_{y}^{+}(z)=\left[\begin{array}{ll}
\beta_{y}^{T}(z) & \Delta C_{y}(z)
\end{array}\right]^{T} ; \\
& \theta_{r}^{+}=\left[\begin{array}{ll}
\theta_{r}^{T} & \vartheta_{r}^{n_{r}+1}
\end{array}\right]^{T} ; \quad \theta_{y}^{+}=\left[\begin{array}{ll}
\theta_{y}^{T} & \vartheta_{y}^{n_{y}+1}
\end{array}\right]^{T}
\end{aligned}
$$


Consider now the extended performance index

$$
J_{M R}^{+}\left(\theta_{r}^{+}, \theta_{y}^{+}\right)=\left\|\left(\frac{P(z) C_{r}^{+}\left(z ; \theta_{r}^{+}\right)}{1+P(z) C_{y}^{+}\left(z ; \theta_{y}^{+}\right)}-M(z)\right) W_{M}(z)\right\|_{2}^{2}+\left\|\left(\frac{1}{1+P(z) C_{y}^{+}\left(z ; \theta_{y}^{+}\right)}-S(z)\right) W_{S}(z)\right\|_{2}^{2}
$$

Clearly, the global minimizer $\left(\bar{\theta}_{r}^{+}, \bar{\theta}_{y}^{+}\right)$of $J_{M R}^{+}\left(\theta_{r}^{+}, \theta_{y}^{+}\right)$is such that $J_{M R}^{+}\left(\bar{\theta}_{r}^{+}, \bar{\theta}_{y}^{+}\right)=0$. Moreover, $C_{r}^{+}\left(z ; \bar{\theta}_{r}^{+}\right)=C_{r}^{0}(z), C_{y}^{+}\left(z ; \bar{\theta}_{y}^{+}\right)=C_{y}^{0}(z)$. Letting $\bar{J}_{M R}^{+}\left(\theta_{r}^{+}, \theta_{y}^{+}\right)$be the second order Taylor expansion of $J_{M R}^{+}\left(\theta_{r}^{+}, \theta_{y}^{+}\right)$around its global minimizer $\left(\bar{\theta}_{r}^{+}, \bar{\theta}_{y}^{+}\right)$, we have now the following theorem.

Theorem 2.1 The minimizer $\left(\hat{\theta}_{r}, \hat{\theta}_{y}\right)$ of the performance index $J_{V R}\left(\theta_{r}, \theta_{y}\right)$ where the filters $L_{M}(z)$ and $L_{S}(z)$ have been selected according to (10) and (11) is given by:

$$
\left(\hat{\theta}_{r}, \hat{\theta}_{y}\right)=\arg \min _{\left(\theta_{r}, \theta_{y}\right)} \bar{J}_{M R}^{+}\left(\left[\begin{array}{ll}
\theta_{r}^{T} & 0
\end{array}\right]^{T},\left[\begin{array}{ll}
\theta_{y}^{T} & 0
\end{array}\right]^{T}\right)
$$

Before proving the theorem, some remarks about its interpretation are in order.

The theorem states that $\left(\hat{\theta}_{r}, \hat{\theta}_{y}\right)$ minimizes the restriction to the admissible controller parameter subspace of the second order expansion of the extended model reference performance index around its minimizer. On the other hand, the optimal controller parameter $\left(\bar{\theta}_{r}, \bar{\theta}_{y}\right)$ minimizes the restriction to the admissible controller parameter subspace of the extended model reference performance index. Thus, we see that the difference between $\left(\hat{\theta}_{r}, \hat{\theta}_{y}\right)$ and $\left(\bar{\theta}_{r}, \bar{\theta}_{y}\right)$ rests on the fact that the former is obtained from a simplified quadratic version of the performance index that is minimized by the latter.

Note now that a very important step in the solution of the control problem design is a sensible selection of the controller class. On the one hand, the controller class should be as simple as possible. On the other hand, it need be rich enough so that the best controller within the class attains a satisfactory performance. Discussing the way a sensible controller class can be selected goes beyond the scope of the present paper. Here, we want to observe that, if a sensible selection has been performed, then the role played by $\Delta C_{r}(z)$ and $\Delta C_{y}(z)$ in the determination of $\left(C_{r}^{0}(z), C_{y}^{0}(z)\right)$ is only marginal. As a consequence, $\left(\hat{\theta}_{r}, \hat{\theta}_{y}\right)$ is a good approximation to $\left(\bar{\theta}_{r}, \bar{\theta}_{y}\right)$ since $J_{M R}^{+}\left(\theta_{r}^{+}, \theta_{y}^{+}\right)$ is well approximated in a neighborhood of its minimum by its second order expansion $\bar{J}_{M R}^{+}\left(\theta_{r}^{+}, \theta_{y}^{+}\right)$.

Proof of Theorem 2.1: Since $\bar{\theta}^{+}$minimizes $\bar{J}_{M R}^{+}$and attains perfect matching, the second order expansion only consists of the second order term. Simple, though cumbersome, computations then show that ( ${ }^{-}$denotes complex conjugation) $\bar{J}_{M R}^{+}\left(\theta_{r}^{+}, \theta_{y}^{+}\right)$has the following expression 


$$
\begin{aligned}
\bar{J}_{M R}^{+}\left(\theta_{r}^{+}, \theta_{y}^{+}\right) & \\
= & {\left[\begin{array}{c}
\theta_{r}^{+}-\bar{\theta}_{r}^{+} \\
\theta_{y}^{+}-\bar{\theta}_{y}^{+}
\end{array}\right]^{T}\left(\frac{1}{2 \pi} \int_{-\pi}^{\pi}|P|^{2}\left[\begin{array}{cc}
\overline{\beta_{r}^{+}} \beta_{r}^{+T} & -M \overline{\beta_{r}^{+}} \beta_{y}^{+T} \\
-\overline{M \beta_{y}^{+}} \beta_{r}^{+T} & |M|^{2} \overline{\beta_{y}^{+}} \beta_{y}^{+T}
\end{array}\right] \frac{\left|W_{M}\right|^{2}}{\left|1+P C_{y}^{0}\right|^{2}} d \omega\right)\left[\begin{array}{c}
\theta_{r}^{+}-\bar{\theta}_{r}^{+} \\
\theta_{y}^{+}-\bar{\theta}_{y}^{+}
\end{array}\right] } \\
& +\left(\theta_{y}^{+}-\bar{\theta}_{y}^{+}\right)^{T}\left(\frac{1}{2 \pi} \int_{-\pi}^{\pi} \frac{\left.|P|^{2}|S|^{2}\left|W_{S}\right|^{2} \overline{\beta_{y}^{+}} \beta_{y}^{+T} d \omega\right)\left(\theta_{y}^{+}-\bar{\theta}_{y}^{+}\right)}{\left|1+P C_{y}^{0}\right|^{2}}\right. \\
= & \frac{1}{2 \pi} \int_{-\pi}^{\pi}|P|^{2}\left|C_{r}^{0}-C_{r}^{+}\left(\theta_{r}^{+}\right)+M\left(C_{y}^{+}\left(\theta_{y}^{+}\right)-C_{y}^{0}\right)\right|^{2} \frac{\left|W_{M}\right|^{2}}{\left|1+P C_{y}^{0}\right|^{2}} d \omega \\
& +\frac{1}{2 \pi} \int_{-\pi}^{\pi}|P|^{2}\left|C_{y}^{+}\left(\theta_{y}^{+}\right)-C_{y}^{0}\right|^{2} \frac{|S|^{2}\left|W_{S}\right|^{2}}{\left|1+P C_{y}^{0}\right|^{2}} d \omega
\end{aligned}
$$

Consider now the cost function $J_{V R}\left(\theta_{r}, \theta_{y}\right)$. If the filters $L_{M}(z), L_{S}(z)$ are selected as in (10) and (11), then $J_{V R}\left(\theta_{r}, \theta_{y}\right)$ is given by:

$$
\begin{aligned}
J_{V R}\left(\theta_{r}, \theta_{y}\right)= & \frac{1}{2 \pi} \int_{-\pi}^{\pi}|P|^{2}\left|C_{r}^{0}-C_{r}\left(\theta_{r}\right)+M\left(C_{y}\left(\theta_{y}\right)-C_{y}^{0}\right)\right|^{2} \frac{\left|W_{M}\right|^{2}}{\left|1+P C_{y}^{0}\right|^{2}} \Phi_{u} \\
& +\frac{1}{2 \pi} \int_{-\pi}^{\pi}|P|^{2}\left|C_{y}\left(\theta_{y}\right)-C_{y}^{0}\right|^{2} \frac{|S|^{2}\left|W_{S}\right|^{2}}{\left|1+P C_{y}^{0}\right|^{2}} \Phi_{u} d \omega
\end{aligned}
$$

By comparing (13) and (14), we note that $\left.J_{V R}\left(\theta_{r}, \theta_{y}\right)=\bar{J}_{M R}^{+}\left(\begin{array}{ll}\theta_{r}^{T} & 0\end{array}\right]^{T},\left[\begin{array}{ll}\theta_{y}^{T} & 0\end{array}\right]^{T}\right)$, from which (12) follows.

\section{Inclusion of a fixed integral action}

It is a standard requirement that the control system guarantees, in steady state, null tracking error for constant references and perfect rejection of constant disturbances. This is expressed through the static gains of the reference models as: $M(1)=1$ (for tracking) and $S(1)=0$ (for disturbance rejection).

The correct static gains can be rigorously imposed by introducing an integral action in the controllers, that is by taking

$$
C_{r}\left(z ; \theta_{r}\right)=\frac{1}{1-z^{-1}} C_{r}^{\prime}\left(z ; \theta_{r}\right), \quad C_{y}\left(z ; \theta_{y}\right)=\frac{1}{1-z^{-1}} C_{y}^{\prime}\left(z ; \theta_{y}\right)
$$

(in the implementation of the controller, $\frac{1}{1-z^{-1}}$ will be placed in the loop), and by further imposing that $C_{r}^{\prime}\left(z ; \theta_{r}\right)$ and $C_{y}^{\prime}\left(z ; \theta_{y}\right)$ have the same static gain, i.e. $C_{r}^{\prime}\left(1 ; \theta_{r}\right)=C_{y}^{\prime}\left(1 ; \theta_{y}\right)$.

If the controller is linearly parameterized as follows

$$
C_{r}^{\prime}\left(z ; \theta_{r}\right)=\beta_{r}^{\prime}(z)^{T} \theta_{r} ; \quad C_{y}^{\prime}\left(z ; \theta_{y}\right)=\beta_{y}^{\prime}(z)^{T} \theta_{y}
$$


the condition $C_{r}^{\prime}\left(1 ; \theta_{r}\right)=C_{y}^{\prime}\left(1 ; \theta_{y}\right)$ is expressed as:

$$
v^{T}\left[\begin{array}{ll}
\theta_{r}^{T} & \theta_{y}^{T}
\end{array}\right]^{T}=0, \quad v=\left[\beta_{r}^{\prime}(1)^{T} \quad-\beta_{y}^{\prime}(1)^{T}\right]^{T}
$$

Since (15) is a linear constraint, the constrained minimum of $J_{V R}^{N}\left(\theta_{r}, \theta_{y}\right)$ is easily achieved by imposing orthogonality between the gradient of $J_{V R}^{N}\left(\theta_{r}, \theta_{y}\right)$ and the subspace of the feasible parameter vectors. This is obtained by solving the following set of equations with respect to $\left(\theta_{r}, \theta_{y}\right)$ and $\lambda$ :

$$
\left\{\begin{array}{l}
{\left[\begin{array}{c}
\frac{d}{d \theta_{r}} J_{V R}^{N}\left(\theta_{r}, \theta_{y}\right) \\
\frac{d}{d \theta_{y}} J_{V R}^{N}\left(\theta_{r}, \theta_{y}\right)
\end{array}\right]=\lambda v} \\
v^{T}\left[\begin{array}{ll}
\theta_{r}^{T} & \theta_{y}^{T}
\end{array}\right]^{T}=0
\end{array}\right.
$$

With the usual meaning of all symbols, the solution is easily seen to be given by

$$
\left[\begin{array}{c}
\hat{\theta}_{r}^{N} \\
\hat{\theta}_{y}^{N}
\end{array}\right]=A_{N}^{-1}\left[F_{N}-\hat{\lambda} v\right], \quad \hat{\lambda}=\frac{v^{T} A_{N}^{-1} F_{N}}{v^{T} A_{N}^{-1} v}
$$

We also note that the result given in Theorem 2.1 holds with minor modifications in the present context: in this case, $\left(\hat{\theta}_{r}, \hat{\theta}_{y}\right)$ is the minimizer of $\left.\bar{J}_{M R}^{+}\left(\begin{array}{ll}\theta_{r}^{T} & 0\end{array}\right]^{T},\left[\begin{array}{ll}\theta_{y}^{T} & 0\end{array}\right]^{T}\right)$ subject to the contraint that the gains of the two controller transfer functions are equal.

\section{The use of noisy data}

The analysis of the previous section has been conducted under the assumption that the system is noise-free. In this section, we turn to consider a more realistic noisy setting.

Let us assume that a set of data $\{u(t), y(t)\}_{t=1, . ., N}$ has been collected from a noisy experiment, that is the system (1) incorporates a nonzero noise term $d(t)$. If the design algorithm of Section 2 is applied, the resulting estimate $\left(\hat{\theta}_{r}^{N}, \hat{\theta}_{y}^{N}\right)$ will be different from what we have seen in the previous section due to the presence of noise and, therefore, the analysis there developed no longer applies. It is perhaps interesting to note that this is true even in the case when the plant operates in openloop. The reason is that in the identification equations (5) the regression vector is constructed from the plant output, so that it is affected by noise.

In $[10,9,24]$, under the hypothesis that $d(t)$ is a stationary stochastic process, an instrumental variable procedure has been proposed in order to counteract the effect of noise in the design of 1 d.o.f. controllers through the virtual reference approach. The extension to 2 d.o.f. design is now sketched. 
Consider an additional set of data $\left\{u(t), y(t)^{\prime}\right\}_{t=1, . ., N}$ collected from a second experiment performed on the plant with the same input signal $\{u(t)\}_{t=1, . ., N}$. Then, estimate the parameter vectors by means of the instrumental variable equations:

$$
\begin{gathered}
{\left[\begin{array}{c}
\hat{\theta}_{r}^{N} \\
\hat{\theta}_{y}^{N}
\end{array}\right]=A_{N}^{-1} F_{N}} \\
A_{N}=\frac{1}{N} \sum_{t=1}^{N}\left(\left[\begin{array}{c}
\zeta_{L_{M}}^{\bar{r}}(t) \\
-\zeta_{L_{M}}^{y}(t)
\end{array}\right]\left[\begin{array}{c}
\varphi_{L_{M}}^{\bar{r}}(t) \\
-\varphi_{L_{M}}^{y}(t)
\end{array}\right]^{T}+\left[\begin{array}{c}
0 \\
\zeta_{L_{S}}^{\bar{y}}(t)
\end{array}\right]\left[\begin{array}{c}
0 \\
\varphi_{L_{S}}^{\bar{y}}(t)
\end{array}\right]\right) \\
F_{N}=\frac{1}{N} \sum_{t=1}^{N}\left(\left[\begin{array}{c}
\zeta_{L_{M}}^{\bar{r}}(t) \\
-\zeta_{L_{M}}^{y}(t)
\end{array}\right] u_{L_{M}}(t)-\left[\begin{array}{c}
0 \\
\zeta_{L_{S}}^{\bar{y}}(t)
\end{array}\right] u_{L_{S}}(t)\right)
\end{gathered}
$$

where $\left\{\varphi_{L_{M}}^{\bar{r}}(t), \varphi_{L_{M}}^{y}(t), \varphi_{L_{S}}^{\bar{y}}(t)\right\}_{t=1, . ., N}$ are constructed as in (5) on the basis of $\{y(t)\}_{t=1, . ., N}$, while $\left\{\zeta_{L_{M}}^{\bar{r}}(t), \zeta_{L_{M}}^{y}(t), \zeta_{L_{S}}^{\bar{y}}(t)\right\}_{t=1, . ., N}$ are constructed in the same way as the corresponding $\left\{\varphi_{L_{M}}^{\bar{r}}(t)\right.$, $\left.\varphi_{L_{M}}^{y}(t), \varphi_{L_{S}}^{\bar{y}}(t)\right\}_{t=1, . ., N}$ but using the output of the second experiment $\left\{y(t)^{\prime}\right\}_{t=1, . ., N}$.

Clearly, $\left\{y(t)^{\prime}\right\}_{t=1, . ., N}$ is different from $\{y(t)\}_{t=1, . ., N}$ since the two sequences are affected by two different realizations of the noise in the two experiments. If we assume, as it is reasonable, that the noise signals in the two experiments are uncorrelated, then asymptotically (17) returns the same estimate as the one we achieve with the standard normal equations in the noiseless case (in fact, this convergence result requires the standard technical assumptions of instrumental variable methods, as discussed in many textbooks, see e.g. [27]).

An approximate procedure can be formulated even if the second experiment on the plant is not possible. In this case one can use, as instrumental variables, the simulated output of a model of the plant identified from the set of data $\{u(t), y(t)\}_{t=1, . ., N}$ (synthetic data). If the system operates in open-loop, the identification step amounts to a standard open-loop identification. If the plant is in closed-loop configuration, one can instead conceive to identify the whole closed-loop control system and then directly use the closed-loop identified model to generate the synthetic data.

If this second route is adopted, strictly speaking the virtual reference method is no longer fully direct. It is important to note, however, that the estimated plant is used with the only objective of generating an instrumental variable signal and its actual expression is not directly used to design the controller. This in particular implies that a high order model can be used in the identification of $P(z)$ without affecting the controller complexity. 


\section{A Numerical Example}

A simple numerical example illustrates the procedure developed in the previous sections.

Consider the following discrete-time plant

$$
P(z)=\frac{0.1622 z^{-1}-0.01622 z^{-2}}{1-1.7 z^{-1}+0.8825 z^{-2}}
$$

whose magnitude Bode plot is shown in Fig.4. Of course, in the controller design such a transfer function is unknown and it is introduced here for completeness.

The output of the plant if affected by an additive stochastic disturbance signal $d(t)$ having the following form:

$$
d(t)=\frac{0.3}{1-0.7 z^{-1}} \xi(t)
$$

where $\xi(t)$ is white noise with variance $\sigma_{\xi}^{2}=0.01$.

The objective is to design a 2 d.o.f. controller for the plant $P(z)$. The reference models are

$$
\begin{aligned}
& M(z)=\frac{(1-\alpha) z^{-1}}{1-\alpha z^{-1}} \quad \alpha=0.4 \\
& S(z)=1-\frac{(1-\beta) z^{-1}}{1-\beta z^{-1}} \quad \beta=0.8
\end{aligned}
$$

(see Fig. 4 for their magnitude Bode plots). The weighting functions have been chosen as: $W_{M}(z)=$ $W_{S}(z)=\frac{1}{1-z^{-1}}$ in order to emphasize a good tracking for the step response, and the adopted class of controllers is

$$
\begin{aligned}
& C_{r}\left(z ; \theta_{r}\right)=\frac{\vartheta_{0}^{r}+\vartheta_{1}^{r} z^{-1}+\vartheta_{2}^{r} z^{-2}+\vartheta_{3}^{r} z^{-3}+\vartheta_{4}^{r} z^{-4}}{1-z^{-1}} \\
& C_{y}\left(z ; \theta_{y}\right)=\frac{\vartheta_{0}^{y}+\vartheta_{1}^{y} z^{-1}+\vartheta_{2}^{y} z^{-2}+\vartheta_{3}^{y} z^{-3}+\vartheta_{4}^{y} z^{-4}}{1-z^{-1}}
\end{aligned}
$$

A set of open-loop noisy data $\{u(t), y(t)\}_{t=1, . ., 512}$ has been collected by feeding the plant with a white noise signal $\left(\Phi_{u}=1\right)$ and the controller parameters have been estimated through the virtual reference method with the optimal filters (10) and (11). A set of data, obtained by repeating the experiment on the plant with the same input, has been used in order to construct the instrumental variables (see Section 3); moreover, a constrained minimization (16) has been performed to enforce unitary gain of the closed-loop transfer function.

The estimated parameter vectors were:

$$
\begin{aligned}
& \hat{\theta}_{r}^{512}=\left[\begin{array}{llllll}
3.7615 & -7.3513 & 3.6172 & 1.4273 & -1.2026
\end{array}\right]^{T} \\
& \hat{\theta}_{y}^{512}=\left[\begin{array}{llllll}
1.2543 & -2.2753 & 1.6674 & -0.6829 & 0.2887
\end{array}\right]^{T}
\end{aligned}
$$


The performance achieved by the designed control system is illustrated in Fig.5 and Fig.6. In Fig.5, the magnitude Bode plots of the I/O transfer function and of the sensitivity transfer function of the achieved control system are compared with those of the reference models. Fig.6 displays the step response of the designed closed-loop and sensitivity transfer functions.

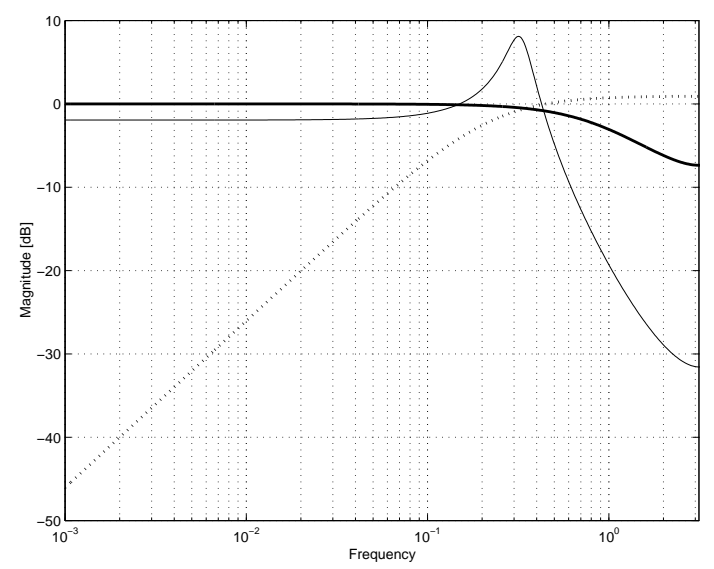

Figure 4: Magnitude Bode plots: The plant (continuous line), the reference-model $M(z)$ (bold line) and the reference sensitivity model $S(z)$ (dotted line).

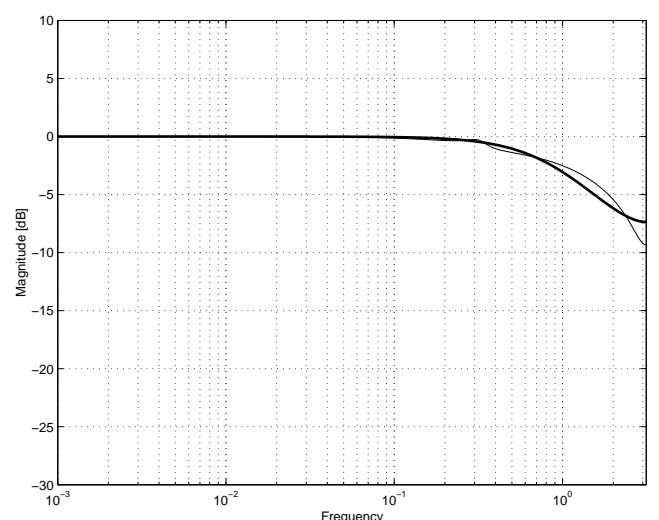

(a)

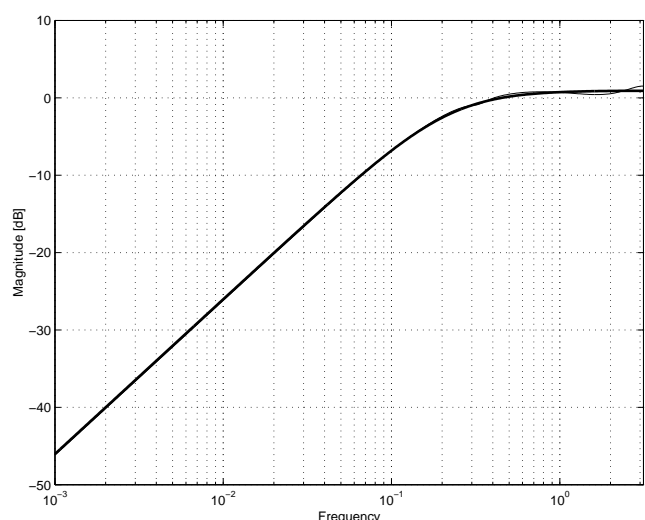

(b)

Figure 5: Magnitude Bode plots. (a) the achieved closed-loop transfer function (thin line) and the referencemodel $M(z)$ (bold line); (b) the achieved sensitivity transfer function (thin line) and the reference-model $S(z)$ (bold line).

\section{Concluding remarks}

In this paper we have presented the extension of the VRFT method to a 2 d.o.f. setting. It has been shown that VRFT is an effective way to directly design from data both the closed-loop transfer function and the sensitivity function. Moreover, VRFT allows for the easy introduction of an integral action in the loop. 


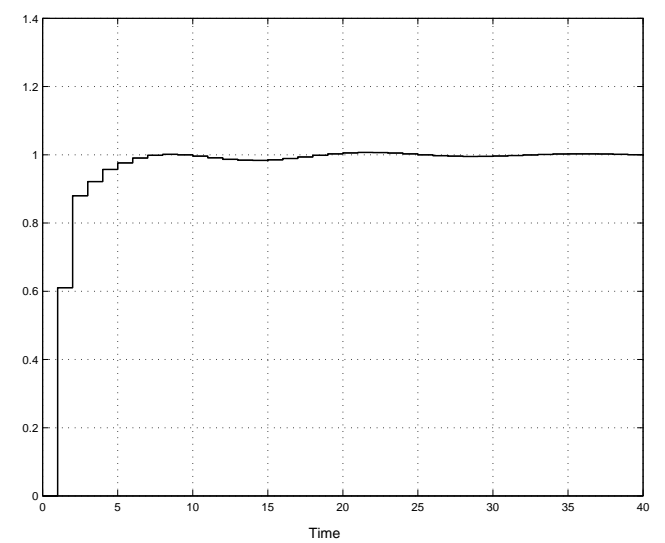

(a)

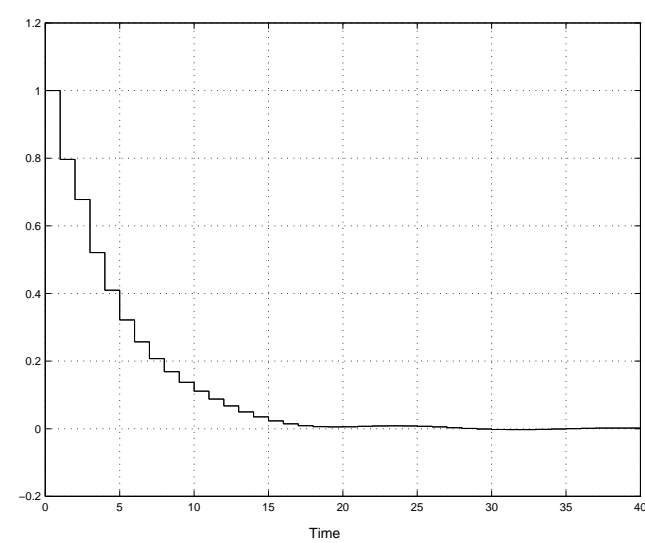

(b)

Figure 6: (a) Step response of the designed closed-loop transfer function; (b) Step response of the designed sensitivity transfer function.

Currently, the use of VRFT for nonlinear plants is under study. The nonlinear setting is very important for applications, since it appears that no easy-to-use one-shot direct methods are available in this context.

Though a discussion of how VRFT extends to nonlinear control problems goes beyod the scope of the present paper, it is perhaps worth mentioning that the main idea underlying VRFT can be applied verbatim in a nonlinear setting. What is lost, however, is the frequency domain interpretation and, with it, an easy way to design suitable data prefilters. 


\section{References}

[1] B.D.O. Anderson and R.L. Kosut. Adaptive robust control: on-line learning. In Proc. of the 30th IEEE Conference on Decision and Control, 297-298, 1991.

[2] K.J. Åström and T. Hägglund. PID Controllers. Theory, Design and Tuning. Instruments Society of America, 1995.

[3] K.J. Åström and B. Wittenmark. Computer-Controlled Systems. Prentice Hall, 1997.

[4] R.B. Bitmead. Iterative control design approaches. In Proc. of 12th IFAC World Congress, volume 9, Sydney, 1993.

[5] X. Bombois, M. Gevers, and G. Scorletti. Controller validation based on an identified model. In Proc. of the 38th IEEE Conf. on Decision and Control, Phoenix, Arizona, 2816-2821, 1999.

[6] X. Bombois, M. Gevers, and G. Scorletti. Controller validation for stability and performance based on frequency domain uncertainty region obtained by stochastic embedding. In Proc. of the 39th IEEE Conf. on Decision and Control, Sydney, Australia, 689-694, 2000.

[7] X. Bombois, M. Gevers, G. Scorletti, and B.D.O. Anderson. Controller validation for stability and performance based on an uncertainty region designed from an identified model. CESAME Technical report, 1999.

[8] X. Bombois, M. Gevers, G. Scorletti, and B.D.O. Anderson. Robustness analysis tools for an uncertainty set obtained by prediction error identification. Automatica, 37(10):1629-1636, 2001.

[9] M.C. Campi, A. Lecchini, and S.M. Savaresi. Virtual Reference Feedback Tuning (VRFT): a new direct approach to the design of feedback controllers. In Proc. of the 39th IEEE Conference on Decision and Control, Sydney, Australia, 623-628, Dec. 2000.

[10] M.C. Campi, A. Lecchini, and S.M. Savaresi. Virtual Reference Feedback Tuning: a direct method for the design of feedback controllers. Automatica, to appear, 2002.

[11] M.C. Campi, A. Lecchini, and S.M. Savaresi. Controller validation: a probabilistic approach based on prediction error identification. preprint, 2002.

[12] K.L. Chien, J.A. Hrones, and Reswick J.B. On the automatic control of generalized passive systems. Trans. ASME, 74:175-185, 1952.

[13] E.B. Dahlin. Designing and tuning digital controllers. Instruments and Control Systems, 42:77-83, June 1968. 
[14] R.A. De Callafon and P.M.J. Van den Hof. Suboptimal feedback control by a scheme of iterative identification and control design. Mathematical Modeling of Systems, 3(1):77-101, 1997.

[15] M. Gevers. Towards a joint design of identification and control? In Essays on Control: Perspectives in the Theory and its Applications, 1735-1740, eds. H.L. Trentekman and C. Willems, 1993.

[16] M. Gevers. Identification for control. In Proc. of the 5th IFAC Symposium on Adaptive Control and Signal Processing, 1-12, 1995.

[17] G.O. Guardabassi and S.M. Savaresi. Virtual Reference Direct Design Method: an off-line approach to data-based control system design. IEEE Trans. Automatic Control, 45(5):954$959,2000$.

[18] G.O Guardabassi and S.M. Savaresi. Approximate linearization via feedback - an overview. Automatica, 37(1):1-15, 2001.

[19] A. Haalman. Adjusting controllers for a dead-time process. Control Engineering, 71-73, July 1965.

[20] R.G. Hakvoort, R. Schrama, and P.M.J. Van den Hof. Approximate identification with closedloop performance criterion and application to LQG feedback design. Automatica, 30(4):679690, 1994.

[21] H. Hjalmarsson. Efficient tuning of linear multivariable controllers using Iterative Feedback Tuning. Int. J. of Adaptive Control and Signal procesing, 13:553-572,1999.

[22] H. Hjalmarsson, M. Gevers, S. Gunnarson, and O. Lequin. Iterative Feedback Tuning: theory and applications. IEEE Control Systems Magazine, 18(4):26-41, 1998.

[23] H. Hjalmarsson, S. Gunnarson, and M. Gevers. A convergent iterative restricted complexity control design scheme. In Proc. of the 33th IEEE Conference on Decision and Control, 17351740, Lake Buona Vista, FL, Dec. 1994.

[24] A. Lecchini. Virtual Reference Feedback Tuning: a new direct data-based method for the design of feedback controllers. PhD Thesis - Università di Brescia, 2001.

[25] W.S. Lee, B.D.O. Anderson, R.L. Kosut, and I.M.Y. Mareels. A new approach to adaptive robust control. Int. J. Adaptive Control and Signal Processing, 7:183-211, 1993.

[26] W.S. Lee, B.D.O. Anderson, R.L. Kosut, and I.M.Y. Mareels. On robust performance improvement through the windsurfer approach to adaptive robust control. In Proc. of the 32th IEEE Conference on Decision and Control, 2821-2827, 1993. 
[27] L. Ljung. System Identification: theory for the user. Prentice Hall, 1999.

[28] G.K. McMillan. Tuning and Control Loop Performance. Instruments Society of America, 1983.

[29] S.M. Savaresi and G.O. Guardabassi. Approximate feedback linearization of discrete time non-linear systems using virtual input direct design. System \& Control Letters, 32:63-67, 1997.

[30] S.M. Savaresi and G.O. Guardabassi. Approximate I/O feedback linearization of discrete-time non-linear systems via virtual input direct design. Automatica, 34(6):715-722, 1998.

[31] R.J. Schrama. Accurate models for control design: the necessity of an iterative scheme. IEEE Trans. Automatic Control, 37(7):991-994, 1992.

[32] J.C. Spall and J.A. Criston. Model-free control of nonlinear stochastic systems with discretetime measurements. IEEE Trans. Automatic Control, 43(9):1198-1210, 1998.

[33] P.M.J. Van den Hof and R. Schrama. Identification and Control - closed loop issues. Automatica, 31(12):1751-1770, 1995.

[34] Z. Zang, R.R. Bitmead, and M. Gevers. Iterative weighted least-squares identification and weighted LQG control. Automatica, 31(11):1577-1594, 1995.

[35] J.G. Ziegler and N.B. Nichols. Optimum settings for automatic controllers. Trans. ASME, 64:759-768, 1942. 